\title{
Leucine Zipper Putative Tumor Suppressor 1
}

National Cancer Institute

\section{Source}

National Cancer Institute. Leucine Zipper Putative Tumor Suppressor 1. NCI Thesaurus. Code C118979.

Leucine zipper putative tumor suppressor 1 (596 aa, $67 \mathrm{kDa}$ ) is encoded by the human LZTS1 gene. This protein plays a role in tumor suppression, cell cycle modulation, cell growth control and transcriptional regulation. 